\title{
Programmed death-ligand 1 monoclonal antibody-linked immunoliposomes for synergistic efficacy of miR-130a and oxaliplatin in gastric cancers
}

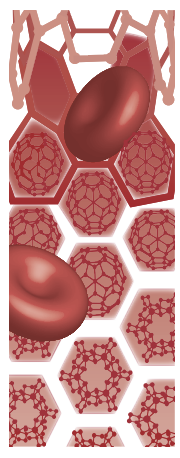

Fengyong Wang ${ }^{1}$, Yuanshui Sun ${ }^{1}$ \& Jianfeng Shi*,1

${ }^{1}$ Department of General Surgery, Tongde Hospital of Zhejiang Province, Hangzhou 310012, PR China

*Author for correspondence: JamisonChandlerehn@yahoo.com

Aim: PD-L1 monoclonal antibody-conjugated miR-130a/oxaliplatin-loaded immunoliposomes were constructed for enhanced therapeutic efficacy against gastric cancer. Materials \& methods: The in vitro antitumor efficacy of the immunoliposomes was evaluated by cell viability, cell invasion, cell apoptosis and western blot analysis and in vivo antitumor efficacy was evaluated in a HGC27-bearing tumor xenograft model. Results: The inhibitory role of miR-130a was demonstrated in HGC27 cells by the downregulation of RAB5A and FOCL1 signaling pathways. Consequently, PD-miOXNP exhibited the strongest anticancer activity in vitro compared with any other formulation. PD-miOXNP showed a significantly higher anticancer efficacy in HGC27 tumors with reduced $\mathrm{Ki}^{+} 7^{+}$cells and increased $\mathrm{TUNEL}^{+}$cells for mice group. Conclusion: PD-L1 monoclonal antibody-conjugated immunoliposomes have immense potential to be applied as a next-generation nanomedicine for PD-L1-positive gastric cancers.

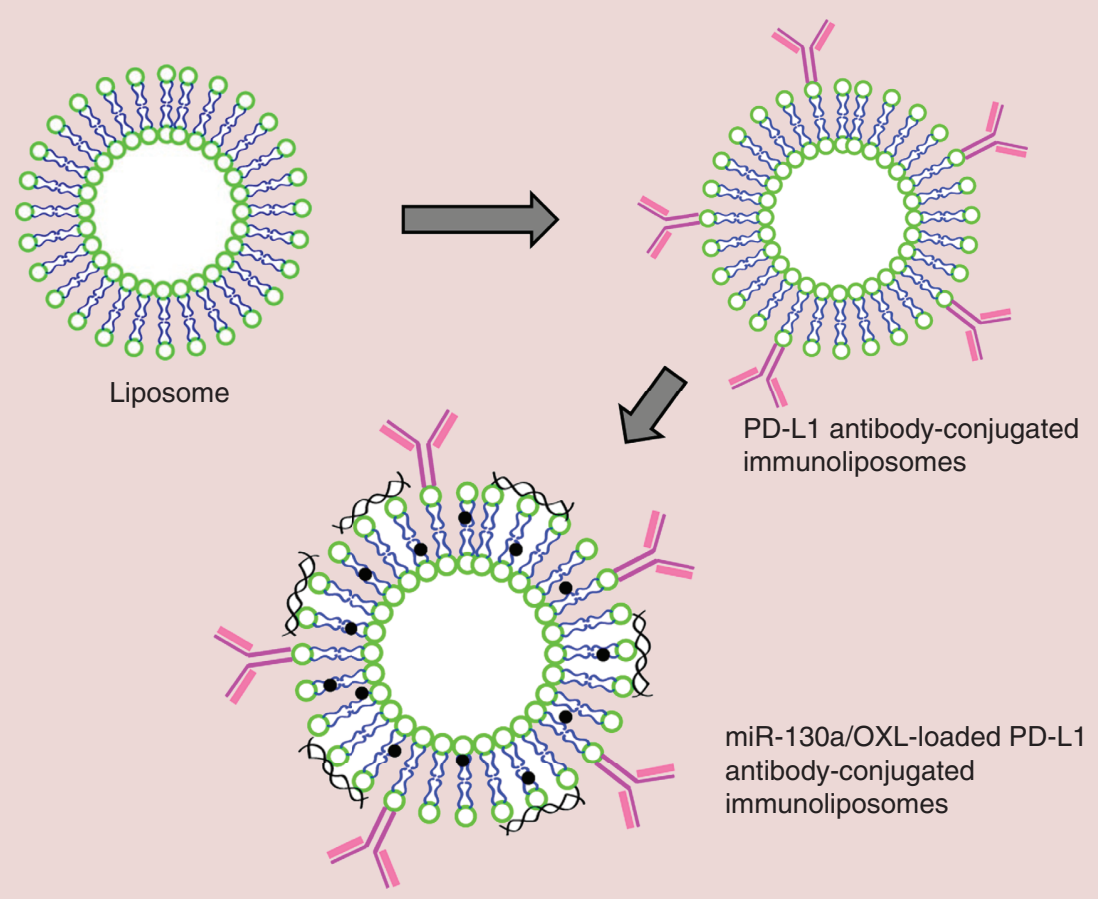

First draft submitted: 20 February 2019; Accepted for publication: 15 April 2019; Published online: 10 July 2019 
Gastric cancer (GC) is the fourth most prevalent form of cancer and the second leading cause of cancer-related deaths worldwide [1]. Every year, approximately, 900,000 patients are diagnosed with gastric carcinoma, with an estimated overall 5 -year survival rate of only $15 \%$ due to high rates of metastasis and recurrence [2,3]. GC patients are diagnosed at later stages and complications such as high metastasis, resistance and relapse make treatment difficult. Present treatment options rely on either chemotherapy or radiation therapy, which are ineffective and lead to poor outcomes $[4,5]$. Nevertheless, chemotherapy drugs, if appropriately used, could be an important tool for the treatment of GCs.

Oxaliplatin (OXL) [oxalato(trans-1,1,2-diaminocyclohexane] platinum(II) (L-OHP) is a third-generation platinum anticancer drug used in the treatment of GC [6]. Although OXL has lower toxicity than cisplatin in the kidney and bone marrow, the tolerable dose of OXL is still limited due to unavoidable adverse effects. The therapeutic utility is also limited due to high levels of erythrocyte binding, which results in systemic toxicity and low concentrations in tumor tissues [7]. At the same time, single-agent-based chemotherapy is increasingly ineffective, necessitating the need for combinational treatments.

miRNAs are a class of small, endogenous, noncoding RNAs (18-22 nucleotides) that are involved in posttranscriptional gene regulation via either repression or degradation of mRNAs [8]. miRNAs have been shown to possess vital roles in carcinogenesis and tumor progression, often serve as either oncogenes or tumor-suppresser genes, and are actively involved in the proliferation, differentiation, invasion, migration and apoptosis of cancer cells [9]. In GC, deregulated expression of miRNAs contributes to carcinogenesis affecting various biological processes and tumor cell growth, and miRNAs may therefore be potential therapeutic targets. For example, miR-21 acts as a potential oncomir and target tumor suppressor genes (PTEN and PDCD4), whereas miR-148a suppresses cancer cell invasion by targeting the ROCK1 gene. In the present study, we used miR-130a to control GC proliferation $[10,11]$. miR-130a has been shown to be involved in the pathogenesis of different cancers, including cervical cancer, ovarian cancer, glioblastoma and leukemia [12-15]. Overexpression of miR-130a targets the $3^{\prime}$-UTR of RAB5A and inhibits the invasion and migration of GC cells. Additionally, miR-130a can target FOSL1, a component of AP-1 transcription factors, which are closely associated with survival and death in GC cells [16]. We have therefore selected miR-130a as a potential oncomir-targeting RAB5A and FOSL1 to improve GC treatment. However, miRNAs are highly unstable in systemic circulation and subject to enzymatic degradation; thus, they need a stable carrier system for systemic or tumor delivery.

Nanotechnology plays an important role in cancer therapy by improving the physicochemical properties of small molecules and the efficient delivery of encapsulated therapeutics [17]. In this regard, liposomes are nontoxic, being composed of nanosized vesicles of a phospholipid bilayer with lipid and aqueous compartments (hydrophilic and hydrophobic derivatives) [18]. The unique quality of liposomes lies in their ability to encapsulate drugs with different physicochemical characteristics, improving pharmacokinetic and biodistribution profiles. Small liposomes of 20-200 nm tend to preferentially accumulate in tumor tissues via enhanced permeability and retention processes [19]. Many targeting moieties such as small-molecule ligands (folate and hyaluronic acid) and peptides (integrin and transferrin) have been used to promote active targeting; however, antibody-conjugated liposomes (i.e., immunoliposomes) are more efficient in targeting tumors [20,21]. Among all antibodies, anti-PD-L1 antibodies have been reported to possess significant clinical benefits in cancer tissues. PD-1 is a cell surface receptor that plays an important role in the immune system, and it also inhibits the inflammatory activity of T-cells [22,23]. PD-1 binds to PD-L1 and transfers an inhibitory signal that decreases the proliferation of antigen-specific T cells [24]. PD-L1 expression has been detected in 40\% of human GCs. A strategy involving the specific binding of PD-L1 antibody to the extracellular domain of PD-L1 in GC may be an effective treatment strategy [25].

Overall, the aim of the present study was to improve anticancer efficiency in GCs by targeting with combinational therapeutics (OXL and miR-130a) using PD-L1-targeted immunoliposomes (Figure 1). OXL and miR-130a-loaded immunoliposomes have been extensively characterized for their physicochemical stabilities. Anticancer efficacy was tested using various in vitro assays. The therapeutic effects of miR-130a on RAB5A and FOSL1 were tested by western blot analysis, and the efficacy was assessed in a HGC23-GC cell-bearing xenograft mice model using immunohistochemical analyses. 


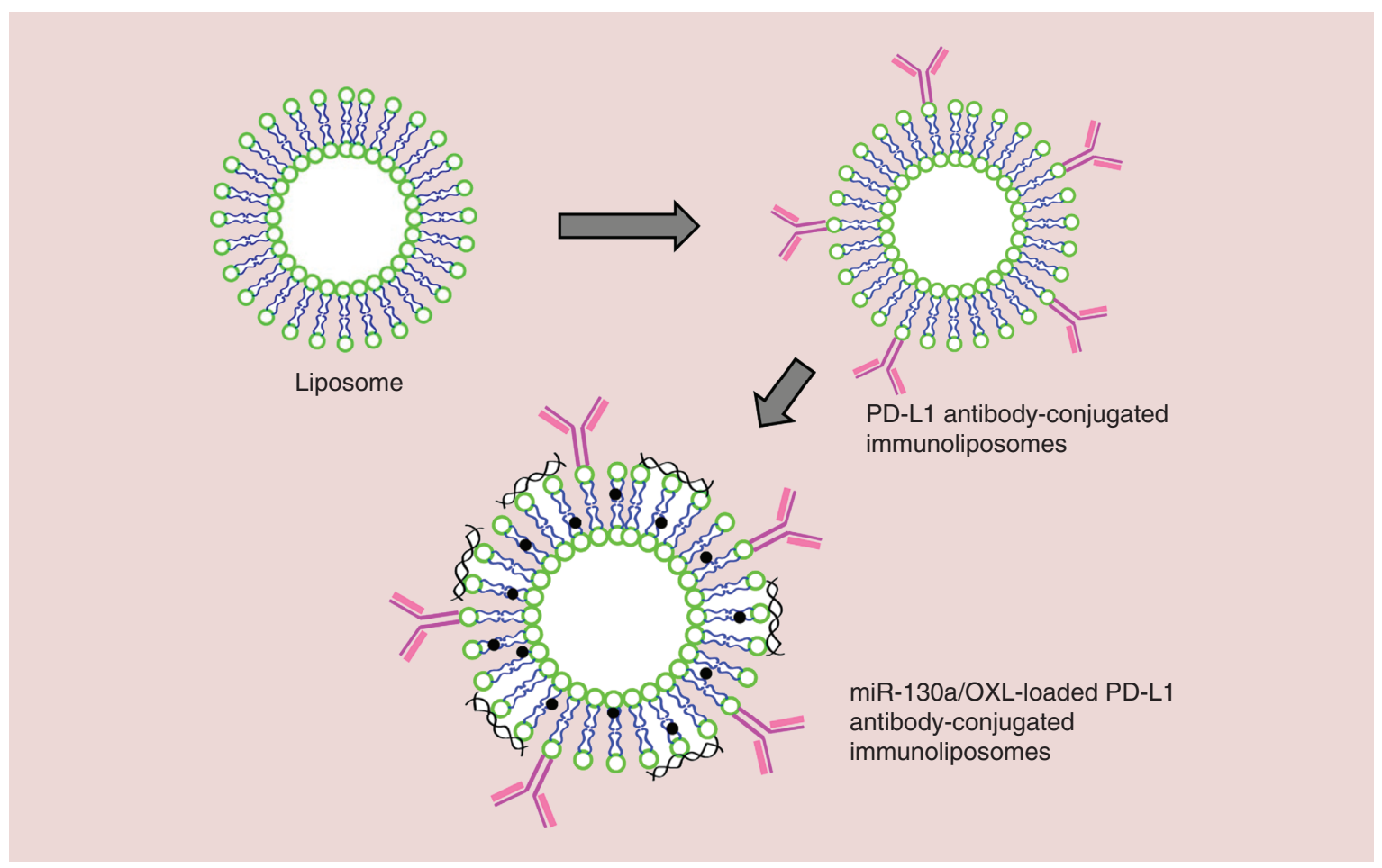

Figure 1. Schematic illustration of preparation of PDL-1 monoclonal antibody-conjugated immunoliposomes. The immunoliposomes were loaded with OXL and miRNA-130a. The OXL is loaded in the lipid bilayer of liposomes and miRNA is electrostatically on the surface of carriers.

OXL: Oxaliplatin.

\section{Materials \& methods}

Preparation of OXL/miRNA-130a-loaded PD-L1-conjuagted immunoliposome

The therapeutic agent-loaded immunoliposome was prepared by thin-film hydration followed by ultrasonication technique. In brief, 1,2-distearoyl-sn-glycero-3-phosphocholine and 1,2-distearoyl-sn-glycero3-phosphoethanolamineN-(methoxy[PEG]-2000; DSPE-PEG2000); cholesterol, DSPE-PEG2000-maleimide (DSPE-PEG2000-Mal) and 1,2-dioleoyl-3-trimethylammonium-propane (chloride salt; Avanti Polar Lipids, China) were dissolved in chloroform at a molar ratio of 4:1:0.5:0.2:0.05. The molar ratio of lipids was selected based on a trial and error basis. Earlier, we have carried out experiments at various molar ratios and optimized the present molar ratio based on particle size and its morphology. The organic solvent was mixed well and OXL (Sigma-Aldrich, China) was added to the mixture and gently stirred. The organic solvents were evaporated using a rotary evaporator followed by vacuum treatment for $3 \mathrm{~h}$ to completely remove all the organic solvent traces. The thin-lipid film was hydrated with $1 \mathrm{X}$ of phosphate-buffered saline (PBS) in $60^{\circ} \mathrm{C}$ for $1 \mathrm{~h}$. The raw lipid suspension was immediately sonicated for 5 min using an ultrasonicator (Probe). The so-formed liposome was centrifuged at $3800 \mathrm{rpm}$ for $10 \mathrm{~min}$ and the supernatant was removed and the OXL-loaded liposome was redispersed in PBS solution. To conjugate the PD-L1 antibody to the liposome surface, liposome and PD-L1 antibody was mixed at $10: 1$ ratio and incubated at $4^{\circ} \mathrm{C}$ for $6 \mathrm{~h}$. The PD-L1 will be conjugated to the liposome using the reaction between sulfhydryl residues on the antibodies to the C-terminal maleimide groups of liposome. The solution was then centrifuged at $12,000 \mathrm{rpm}$ for $15 \mathrm{~min}$ to remove the unconjugated antibody fractions. The supernatant was collected and amount of antibody conjugation was evaluated. The miRNA-130a was incubated with the PD-L1liposomes and incubated for $4 \mathrm{~h}$ at $4^{\circ} \mathrm{C}$. The electrostatic interactions between positively charged liposome and negatively charged miRNA will allow the loading in the liposomes. The unconjugated miRNA was separated by centrifugation at $5000 \mathrm{rpm}$ for $12 \mathrm{~min}$. The supernatant containing the unconjugated miRNA was removed and the pellet was redispersed in buffer. 


\section{Characterization of therapeutics-loaded immunoliposomes}

The hydrodynamic size and surface charge and polydispersity index of immunoliposome was evaluated by NanoZS dynamic light-scattering analyzer (Malvern Instruments, UK). The dispersions were appropriately diluted, and measurements were performed thrice at room temperature. The morphology of particles was evaluated by transmission electron microscope (TEM; H-7600, Hitachi, Japan). The immunoliposomes were dropped in the copper-grid and stained with $2 \%$ phosphotungistic acid and allowed to air dry. The particles were then observed under H-7600 microscope at $100 \mathrm{kV}$. The stability of particles was evaluated in terms of particle size. The immunolipsomes were dispersed in PBS buffer and $10 \%$ serum media. The particles were stored at $4-8^{\circ} \mathrm{C}$ and evaluated for change in particle size throughout the study period until 30 days. The drug-release study was evaluated by dialysis method. The freeze-dried particles containing $2 \mathrm{mg}$ equivalent of OXL was redispersed in $1 \mathrm{ml}$ of release medium (PBS, $\mathrm{pH} 7.4$ ) and acetate-buffered saline ( $\mathrm{pH} 5.0$ ) containing $0.1 \%$ of Tween 80 as a surfactant. $1 \mathrm{ml}$ of dispersion was transferred to a dialysis membrane (MWCO-3500) and immersed in a $25-\mathrm{ml}$ release medium in a centrifugal tube. The tube assembly was placed in a shaker maintained at $100 \mathrm{rpm}$ at $37^{\circ} \mathrm{C}$. The release medium was collected from time to time and the amount of drug released was calculated by HPLC method. HPLC system (Hitachi, Tokyo, Japan) was used with Inertsil C18 column $(150 \times 4.6 \mathrm{~mm}, 5-\mu \mathrm{m}$ particle size, Cosmosil, Nacalai Tesque Inc., CA, USA) as a column. The mobile phase was run at $1 \mathrm{ml}$ per minute at $25^{\circ} \mathrm{C}$.

\section{Gel electrophoresis}

Various concentration of miRNA (N/P ratio) was loaded on the immunoliposome and loaded onto a 2\% Agarose gel (containing $0.5 \%$ ethidium bromide). The gel was run at $80 \mathrm{~V}$ for $30 \mathrm{~min}$. The DNA ladder (Biolabs, MA, USA) was used as a marker and the images were obtained after UV exposure in Gel-Doc system (UVP, Cambridge, UK). Naked miRNA was used as a appropriate control.

\section{Cell culture}

The HGC27 and MGC803 cells were cultured in RPMI 1640 medium supplemented with 10\% FBS and 1\% antibiotic mixture. The cells were grown in ambient conditions of $5 \% \mathrm{CO}_{2}$ and $65 \%$ humidity.

\section{Flow cytometer analysis of PD-L1 expression in GC cells}

The HGC27 and MGC803 cells were harvested and washed thrice with PBS. The $1 \times 10^{6}$ cells were stained with biotinylated monoclonal antibodies (mAbs) to PD-L1. The cells were then incubated with phycoerythrin-labeled streptavidin for $30 \mathrm{~min}$. The cells were then washed with PBS thrice and performed flow cytometer analysis with FACS Calibur flow cytometer. The data and histogram were analyzed using Cell Quest software (BD Biosciences).

\section{Intracellular uptake analysis: confocal laser scanning microscope}

The intracellular uptake of targeted and nontargeted immunoliposomes in HGC27 and MGC803 cells was evaluated by confocal laser scanning microscope. The cells were plated in a 12-well plate and incubated for $24 \mathrm{~h}$. Rhodamine B was used as a fluorescent molecule to track the uptake of the carrier system. The cells were incubated with OXL and miR-130a-loaded lipid nanoparticles (miOXNP) and PD-L1-conjugated OXL (PD-miOXNP) for $1 \mathrm{~h}$. The cells were then washed twice with PBS. The cells were then fixed with $4 \%$ paraformaldehyde and then followed by incubated with 4,6-diamidino-2-phenylindole (blue) for $10 \mathrm{~min}$. The cells were then washed again twice. The cells were mounted on a glass slide and observed under confocal laser scanning microscope (FV1000, Olympus, Japan).

\section{Protein expression analysis by western blotting assay}

The protein expression in cancer cells were determined by western blot analysis. Human gastric cancer (HGC) cells were seeded in a 6-well plate at $2 \times 10^{5}$ cells/well and incubated for $24 \mathrm{~h}$. The cells were treated with miR-130a and scrambled miRNA and incubated for $48 \mathrm{~h}$. The cells were then washed with PBS for three times and harvested in a lysis buffer for $10 \mathrm{~min}$. The cells were centrifuged at 12,000 rpm for $15 \mathrm{~min}$ and supernatant was collected and tested for total protein concentration using bicinchoninic acid assay (BCA) protein assay kit. An equal amount of protein was loaded onto a 10\% SDS-PAGE gel and transferred to a nitrocellulose membrane. The membrane was blocked with $5 \%$ nonfat skim milk for $1 \mathrm{~h}$ to block the nonspecific bindings and then incubated with primary antibodies of RAB5A (Santa Cruz Biotechnology, USA; 1:1000), FOSL1 (Cell Signaling Technology; 1:1000) and GADPH (Cell Signaling Technology; 1:1000), respectively, at $4^{\circ} \mathrm{C}$ overnight. Next day, membranes were washed 
with tween 20/Tris-buffered saline (TBST) and incubated with horseradish peroxidase-labeled goat anti-rabbit or -mouse antibodies (1:3000). The membranes were then visualized with an electrochemiluminescence detection reagent (Beyotime).

\section{Cytotoxicity analysis of miRNA-130a \& synergistic analysis of combinational agents}

The cytotoxicity of free miR-130a and combinational agents (miR-130a and OXL) were determined by MTT assay. The HGC cells were seeded in a 96-well plate at $1 \times 10^{4}$ cells/well and incubated for $24 \mathrm{~h}$. The cells were treated with different concentration of miR-130a, blank nanoparticles (NPs) and different concentrations of formulations (OXL, miOXNP and PD-miOXNP) and incubated for 24 and $48 \mathrm{~h}$, respectively. The media were removed and washed with PBS three-times carefully. The cells were added with $20 \mu \mathrm{l}$ of MTT solution $(5 \mathrm{mg} / \mathrm{ml})$ and incubated for $3 \mathrm{~h}$. The cells were then added with $125 \mu \mathrm{l}$ of DMSO and kept for additional $15 \mathrm{~min}$. The absorbance was read at $570 \mathrm{~nm}$ using a microplate reader (BioTek Instruments Inc., VT, USA). The proportion of cell viability was compared with untreated control.

\section{Cell invasion assay}

The cell invasion assay was performed using Transwell inserts coated with Matrigel matrix. After treatment of cells with respective formulations for $24 \mathrm{~h}$, cells in serum-free media were added to the upper chamber and allowed the invasion to the lower chamber of Transwell, which contains culture media with $10 \%$ FBS and allowed the process for $24 \mathrm{~h}$. After $24 \mathrm{~h}$, noninvading cells were removed from Matrigel and invading cells were fixed with methanol and stained with crystal violet. The cell invasiveness was observed under light microscope at $200 \times$ magnification.

\section{Cell apoptosis assay by flow cytometer \& Hoechst 33342}

The cell apoptosis of cancer cells was determined by flow cytometer. The HGC cells were seeded in 12-well plate at $1 \times 10^{5}$ cells/well and incubated for $24 \mathrm{~h}$. The cells were treated with different concentration of formulations (OXL, miOXNP and PD-miOXNP) and incubated for 24 and $48 \mathrm{~h}$, respectively. The cells were then stained with Annexin V-FITC and propidium iodide (PI), respectively, for $15 \mathrm{~min}$. The cells were made the volume up to $1000 \mu \mathrm{l}$ and studied using a BD FACS flow cytometer. The Hoechst staining was performed in a similar manner in the attached cells in the plate. The cells were fixed and observed under fluorescence microscope for the nuclear degradation.

\section{Antitumor efficacy in xenograft mice model \& immunohistochemical analysis}

5-week old female BALB/c nude mice weighing approximately 20-22 g were obtained from Experimental Animal Center of Tongde Hospital of Zhejiang Province, China. The mice were fed regularly and caged in a specific pathogen-free environment with free access to food and water and an alternating $12 \mathrm{~h}$ light/dark cycle was maintained. All animals and experimental procedures were approved by Animal Care and Use Committee of Tongde Hospital of Zhejiang Province, China. The HGC27 GC cells $\left(1 \times 10^{6}\right)$ were injected into the dorsal flanks of nude mice. The experiments were started when the tumor grew approximately to $75 \mathrm{~mm}^{3}$. The mice were randomly divided into six groups with eight mice in each group. The formulation groups are as follows: nontreated control, blank NP, miRNA-130a, free OXL, miOXNP, PD-miOXNP, respectively. The equivalent OXL concentration was fixed at $7.5 \mathrm{mg} / \mathrm{kg}$ of body mass. The formulations were administered three-times with a gap of 3 days between the formulations. The tumor volume was recorded using Vernier calipers and calculated using $\mathrm{V}=1 / 2 \mathrm{LW}^{2}$, where $\mathrm{L}$ is the longest diameter and $\mathrm{W}$ is the shortest diameter, respectively. All mice were regularly monitored for any discomfort or abnormal posture or breathing problems. All mice were sacrificed under anesthesia and major organs and tumors were collected for histology analysis. The tumors were fixed in $10 \%$ formalin solution and embedded in paraffin. After deparaffinization, tumor tissues were performed using terminal deoxynucleotidyl transferase-mediated nick end labeling (TUNEL) assays and Ki67 assays as per the manufacturer's recommendation. The images were obtained using Olympus equipped with a Hamamatsu Digital Camera ORCA-03 G. Four fields of each section after TUNEL and Ki67 staining were chosen and counted for apoptosis and proliferative cells.

\section{Results}

Design \& characterization of PD-L1-conjugated dual therapeutic-loaded immunoliposomes The average particle size of PD-miOXNP was $168.25 \pm 1.26 \mathrm{~nm}$ with a narrow distribution range of 0.128 (polydispersity index; Figure 2A). The size was again confirmed using transmission electron microscopy (TEM). 


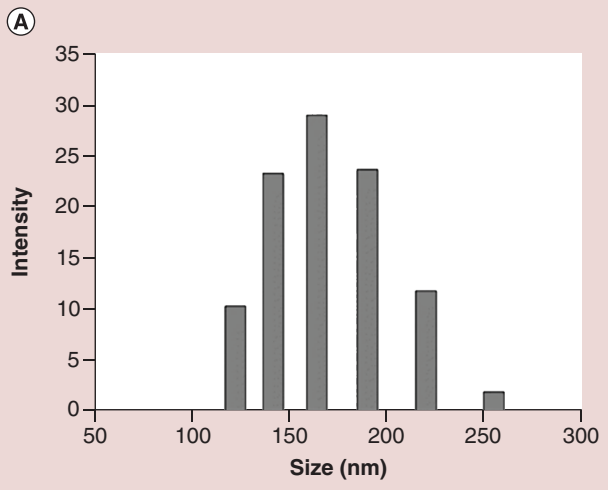

(D)

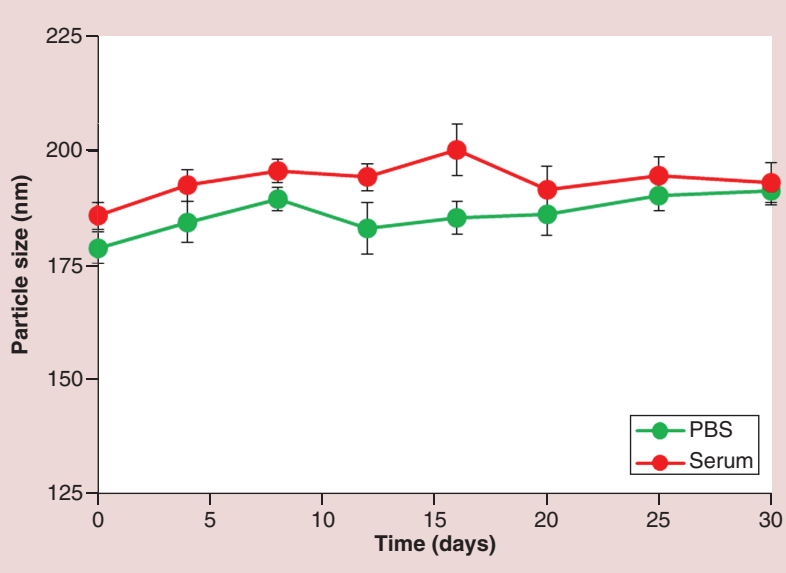

(B)

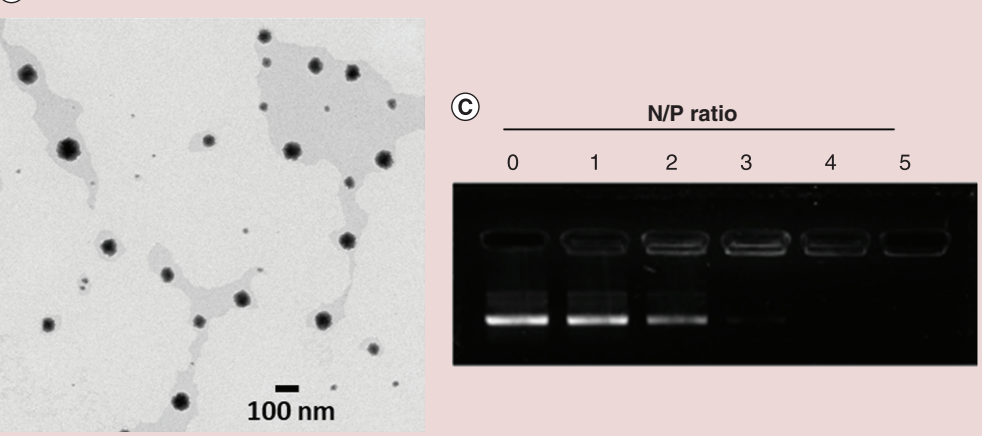

(E)

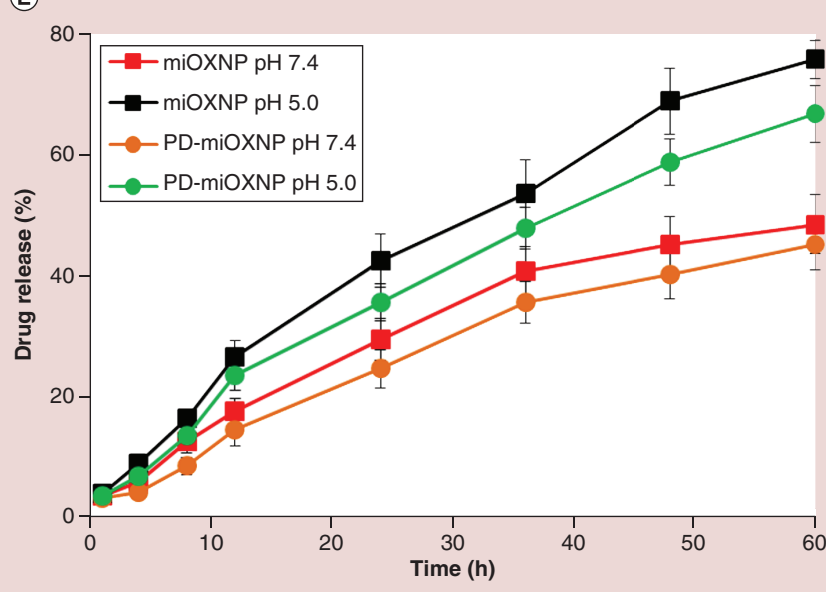

Figure 2. Characterization of PD-L1-conjugated oxaliplatin. (A) Particle size analysis by dynamic light scattering; (B) morphology analysis of PD-miOXNP by transmission electron microscope; (C) gel electrophoresis analysis of miRNA loading in the immunoliposomes; (D) stability analysis of PD-miOXNP in terms of particle size; (E) in vitro drug release profile of OXL from miOXNP and PD-miOXNP in two different $\mathrm{pH}$ conditions (PBS, pH 7.4 and acetate-buffered saline, $\mathrm{pH}$ 5.0).

miOXNP: Oxaliplatin and miR-130a-loaded lipid nanoparticles; N/P: Nitrogen to phosphate; OXL: Oxaliplatin; PBS: Phosphate-buffered saline; PD-miOXNP: PD-L1-conjugated oxaliplatin.

The particles were uniformly spread across the carbon grid and showed a perfect spherical morphology (Figure 2B). It is worth noting that the particle size determined using TEM was smaller than the size determined by differential light scattering. The discrepancy in particle size between TEM and differential light scattering was attributed to the dried state versus aqueous state of the particles. The surface charge of PD-miOXNP was $21.5 \pm 1.45 \mathrm{mV}$, indicating a moderate positive charge sufficient to complex the miRNA on the surface of a carrier system. The high permeability of tumor tissues will reportedly facilitate the accumulation of particles less than $200 \mathrm{~nm}$ via the enhanced permeability and retention effect. The encapsulation efficiency was observed to be $92.5 \pm 2.61 \%$ $(\mathrm{w} / \mathrm{w})$ with an active loading capacity of $9.42 \pm 1.68 \%(\mathrm{w} / \mathrm{w})$. The loading of miRNA was determined by gel electrophoresis. As shown, naked miRNA migrated to the other end without any resistance, whereas PD-miOXNP had a lower mobility with an increase in the N/P ratio (Figure 2C). At an N/P ratio of 4 or 5, 100\% loading of miRNA was observed, indicating successful loading of the immunoliposomes.

\section{Colloidal stability \& drug release}

Physical stability is an important prerequisite for systemic application of a drug delivery system. The particle stability was therefore studied under physiological conditions, and a change in particle size was considered an indicator of instability. The physiological conditions included PBS and culture medium containing 10\% serum. The PDmiOXNP size remained very stable throughout the study period (Figure 2D). Although slight fluctuations were observed at different time points, the particles showed good stability until the end of the study period. The particles maintained stability without being significantly affected by the environment for a fairly long time, indicating their 


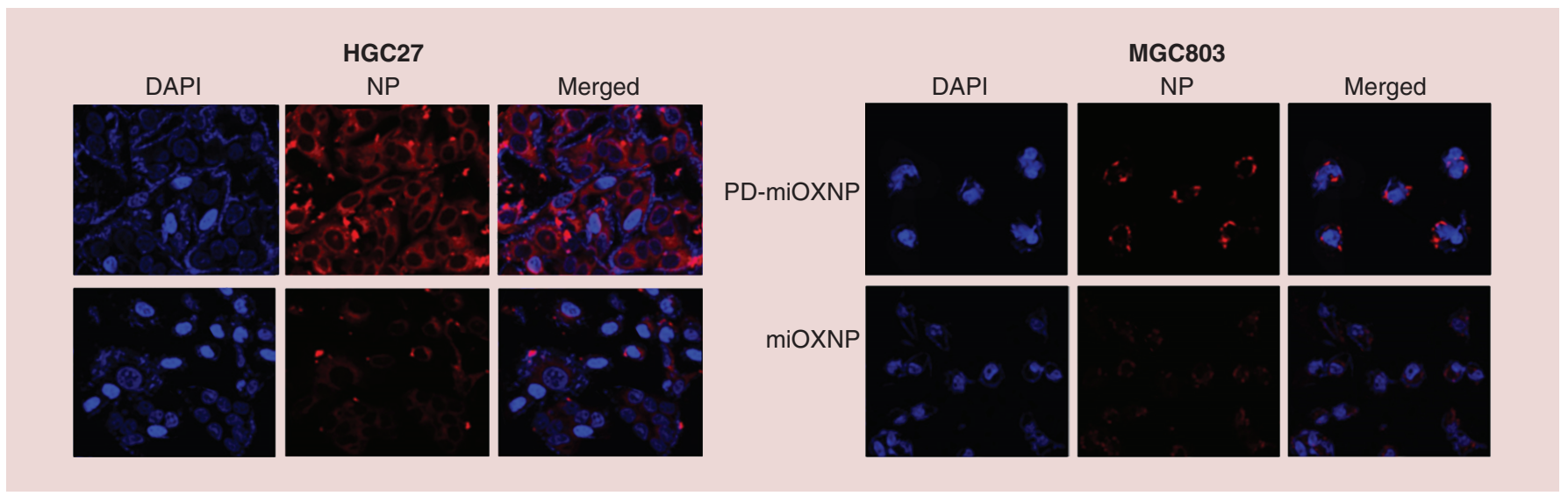

Figure 3. In vitro cellular uptake analysis of oxaliplatin and miR-130a-loaded lipid nanoparticles and PD-L1-conjugated oxaliplatin in HGC27 and MGC803 cancer cells. The cellular uptake of nanoparticles was evaluated by confocal laser scanning microscopy. DAPI: 4',6-diamidino-2-phenylindole; miOXNP: Oxaliplatin and miR-130a-loaded lipid nanoparticles; NP: Nanoparticles; PD-miOXNP: PD-L1-conjugated oxaliplatin.

excellent physical and colloidal stabilities. The in vitro drug release was studied using a dialysis method. As shown, there was a nonsignificant difference in release behavior between miOXNP and PD-miOXNP. The presence of antibody on the surface of NPs did not influence the release of drugs into the medium. For example, approximately $30 \%$ of OXL was released from miOXNP, whereas $26 \%$ of the drug was released from PD-miOXNP after $24 \mathrm{~h}$ (Figure 2E). Nevertheless, a controlled release of drug from PD-miOXNP was observed throughout the study period, with no sign of a burst release pattern, indicating the successful loading of OXL in the immunoliposomes. Moreover, different release behaviors were observed under different $\mathrm{pH}$ conditions. It should be noted that no $\mathrm{pH}$-responsive component was present in the liposome, thus the difference in release behaviors between different $\mathrm{pH}$ conditions may have been due to the faster diffusion of drugs at a lower $\mathrm{pH}$. A quicker release of drug under acidic conditions will enhance the anticancer effects of OXL, whereas a slower or controlled release of drugs at physiological $\mathrm{pH}(\mathrm{pH} 7.4)$ will help reduce systemic side effects [26].

\section{PD-L1 expression and cellular uptake}

As shown, cellular uptake analysis of miOXNP and PD-miOXNP was performed in HGC27 and MGC803 cancer cells (Figure 3). Consistent with the high expression of PD-L1 receptors in HGC27 cells, PD-miOXNP showed remarkable cellular uptake in these cells. A dark red fluorescence (rhodamine B) was observed surrounding the cell cytoplasm, clearly indicating typical receptor-mediated cellular uptake. Nontargeted miOXNP showed remarkably lower cellular uptake compared with that of PD-miOXNP in HGC27 cells. Conversely, cellular uptake of miOXNP and PD-miOXNP was relatively similar in MGC803 cells.

\section{Mechanistic role of miR-130a using western blot analysis}

Western blot analysis was performed to determine the target specificity of miR-130a in GC cells. As shown, miR-130a (lipofectamine complexes) significantly downregulated the expression of FOSL1 protein in GC cells (Figure 4A \& B). The results further confirmed that miR-130a significantly suppressed the expression of RAB4A compared with scrambled miRNA, indicating its target specificity.

\section{In vitro anticancer activities of miR-130a \& PD-miOXNP}

The effects of miR-130a on the viability of HGC27 cancer cells were then evaluated using the MTT assay. As shown, cell viability decreased significantly in a typical concentration-dependent manner with increasing concentrations of miR-130a (Figure 4C).

The influence of a blank nanocarrier was initially tested. As shown, blank NPs did not exhibit any toxic effects in cancer cells, even at the highest concentration tested, indicating the biocompatibility and safety of the blank carrier system (Figure 4D). However, the drug-loaded formulations showed definite anticancer effects on HGC27 cancer cells. As shown, all the formulations exhibited a typical concentration-dependent effect on cancer cells. Treatment 


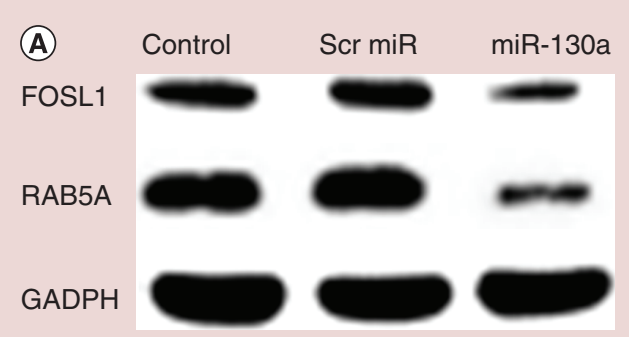

(B)

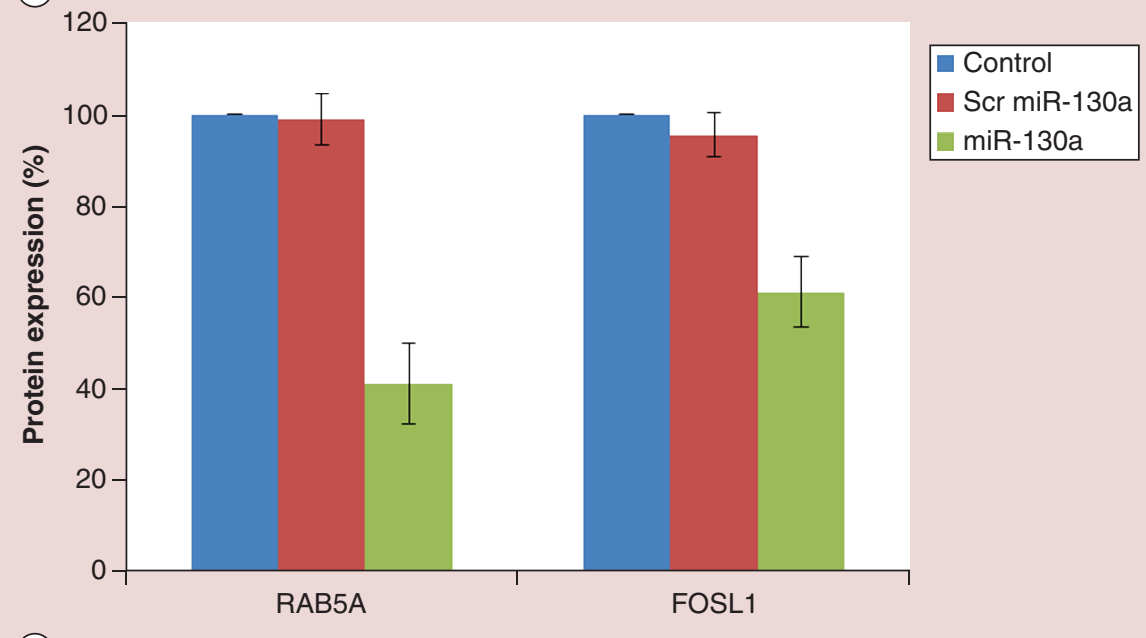

(C)

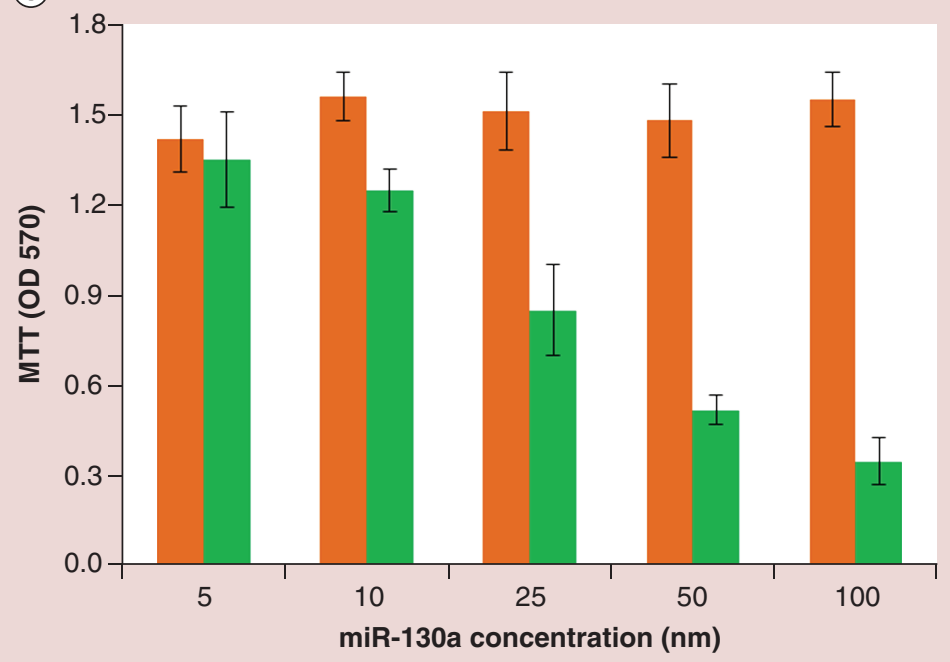

Figure 4. Biological effect of miRNA-130a and oxaliplatin in gastric cancer cells. (A) Targeting effect of miRNA-130a on RAB5 and FOCL1 protein expression by western blot analysis. The HGC27 cells were incubated with miRNA-130a and scrambled miRNA for 48 h; (B) quantification of protein expression by western blot; (C) effect of miRNA-130a on the cell viability of HGC27 cancer cells; (D) effect of blank nanoparticles on the cell viability in a concentration-dependent manner; (E) in vitro cell viability analysis of HGC27 cells upon exposure with different formulations and incubated for $24 \mathrm{~h}$. The cell viability was evaluated by MTT assay. ${ }^{*} p<0.05$ and $* * p<0.01$ are the statistical differences between PD-miOXNP and miOXNP. miOXNP: Oxaliplatin and miR-130a-loaded lipid nanoparticles; OXL: Oxaliplatin; PD-miOXNP: PD-L1-conjugated oxaliplatin; Scr: Scrambled. 
(D)

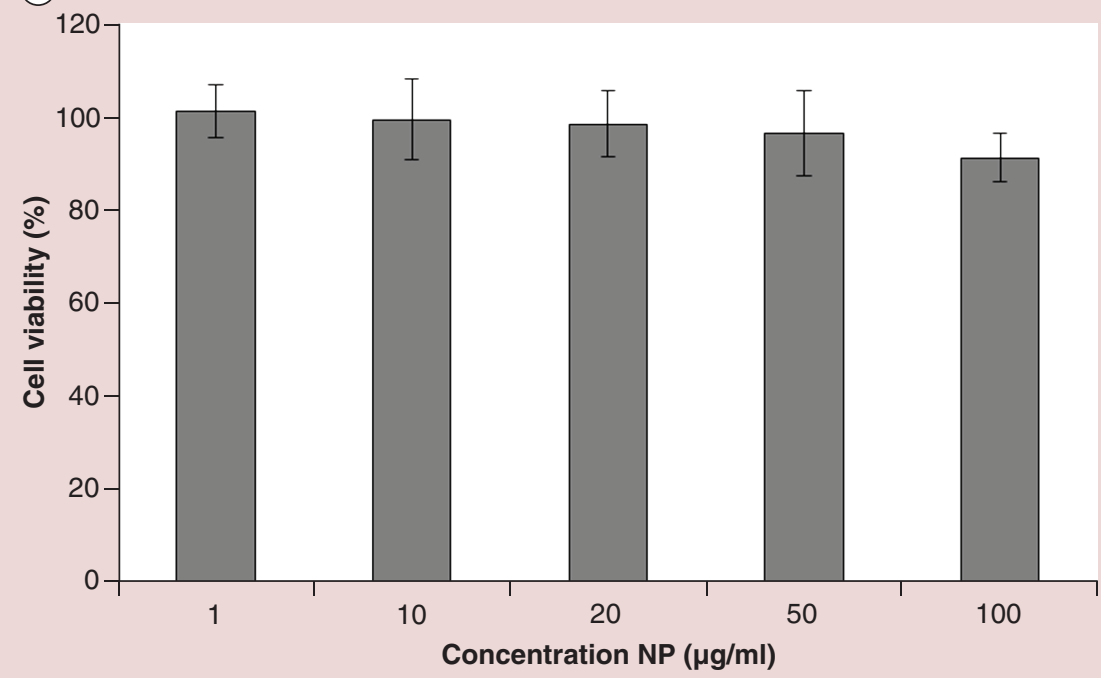

(E)

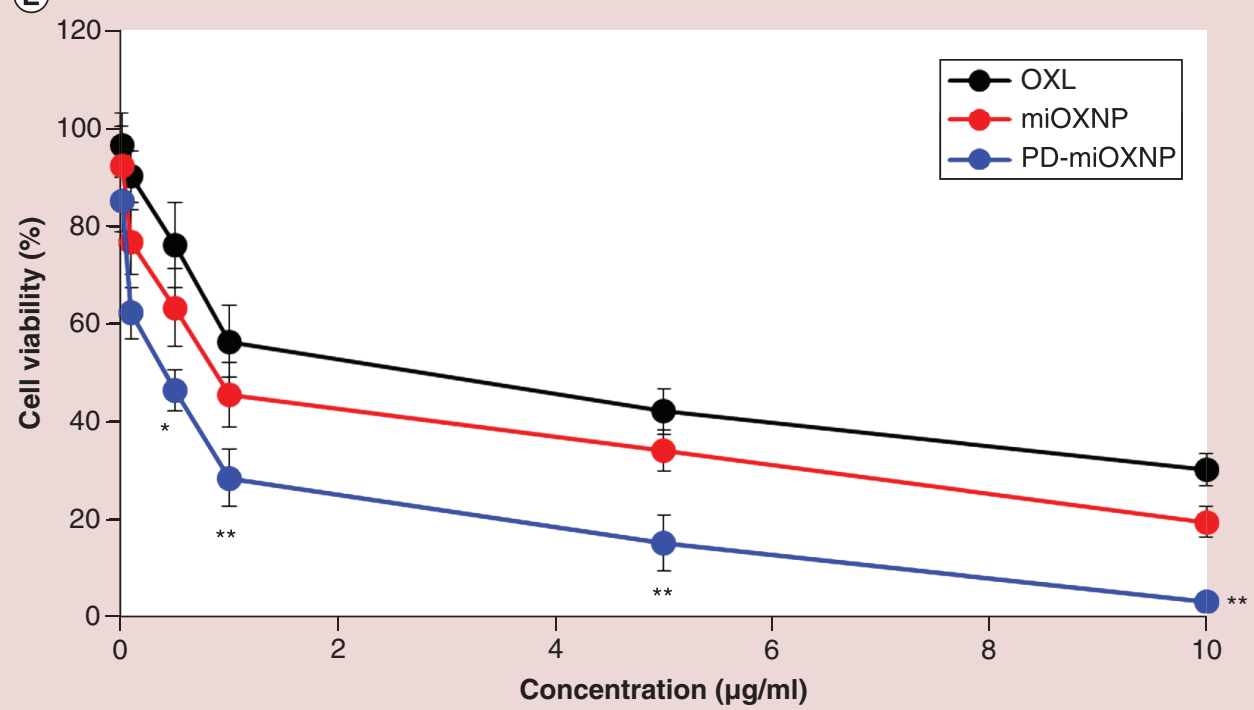

Figure 4. Biological effect of miRNA-130a and oxaliplatin in gastric cancer cells (cont.). (A) Targeting effect of miRNA-130a on RAB5 and FOCL1 protein expression by western blot analysis. The HGC27 cells were incubated with miRNA-130a and scrambled miRNA for 48 h; (B) quantification of protein expression by western blot; (C) effect of miRNA-130a on the cell viability of HGC27 cancer cells; (D) effect of blank nanoparticles on the cell viability in a concentration-dependent manner; (E) in vitro cell viability analysis of HGC27 cells upon exposure with different formulations and incubated for $24 \mathrm{~h}$. The cell viability was evaluated by MTT assay. ${ }^{*} p<0.05$ and ${ }^{* *} p<0.01$ are the statistical differences between PD-miOXNP and miOXNP. miOXNP: Oxaliplatin and miR-130a-loaded lipid nanoparticles; OXL: Oxaliplatin; PD-miOXNP: PD-L1-conjugated oxaliplatin; Scr: Scrambled.

with the combination NPs (miOXNP) resulted in significantly lower cell viability than treatment with the free drug, indicating the synergistic effect of miR-130a and OXL on cancer cells (Figure 4E).

\section{Cellular apoptosis \& cell invasion assays}

The anticancer effects of single and combination agents (with and without targeting) were first evaluated via flow cytometry after staining the cells with annexin-V and propidium iodide (Figure 5A). Consistent with the cell viability assays, miR-130a induced approximately 14\% cell apoptosis, whereas OXL induced $21 \%$ apoptosis. As expected, the combinational regimen (miOXNP) induced higher apoptosis (35\%), whereas PD-miOXNP 
(A)
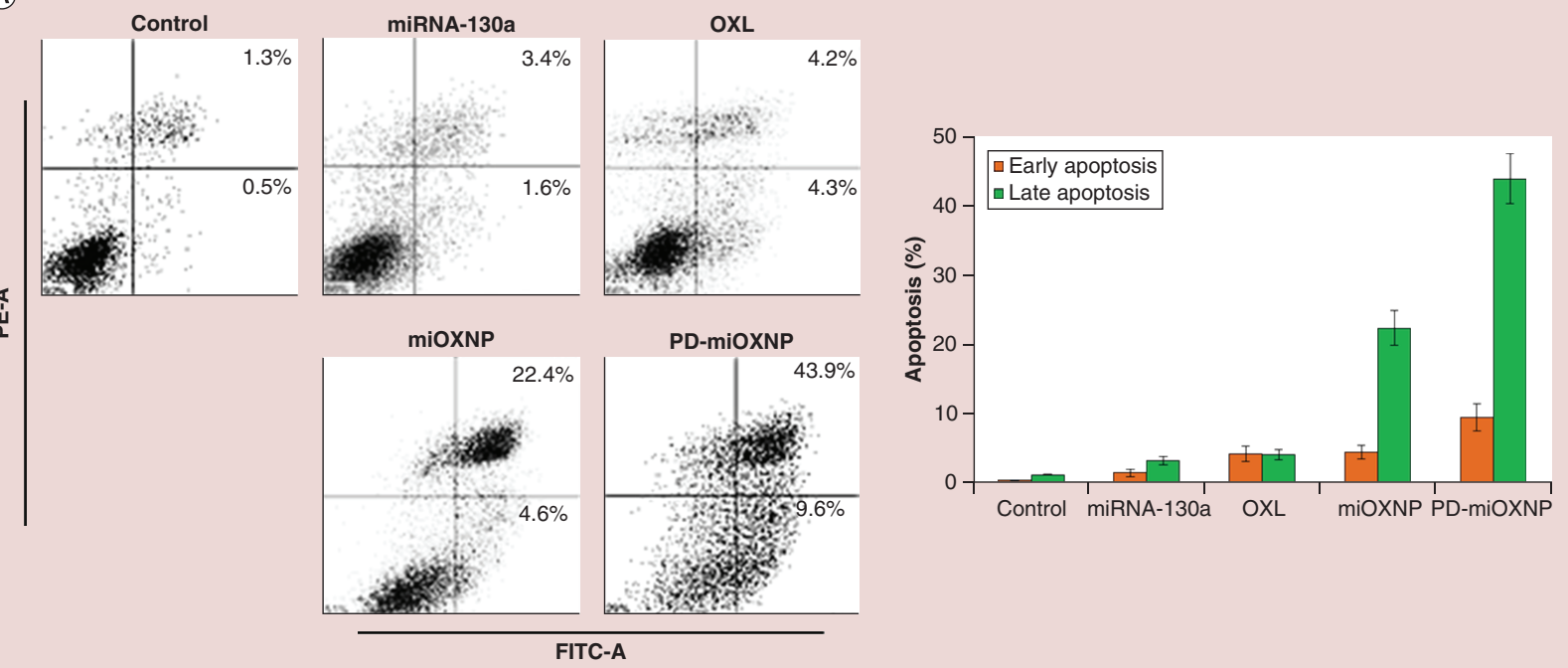

(B)

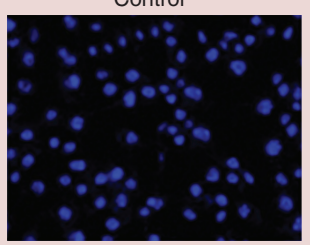

miRNA-130a
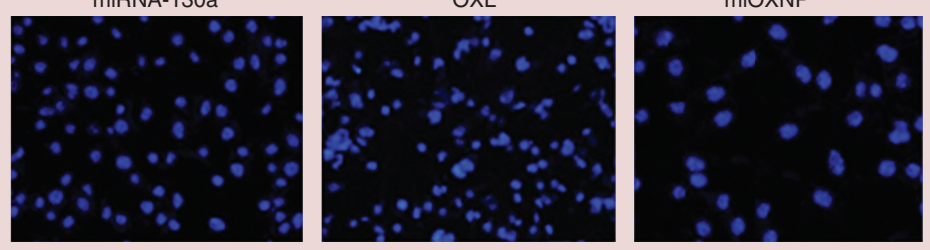

PD-miOXNP

(c)
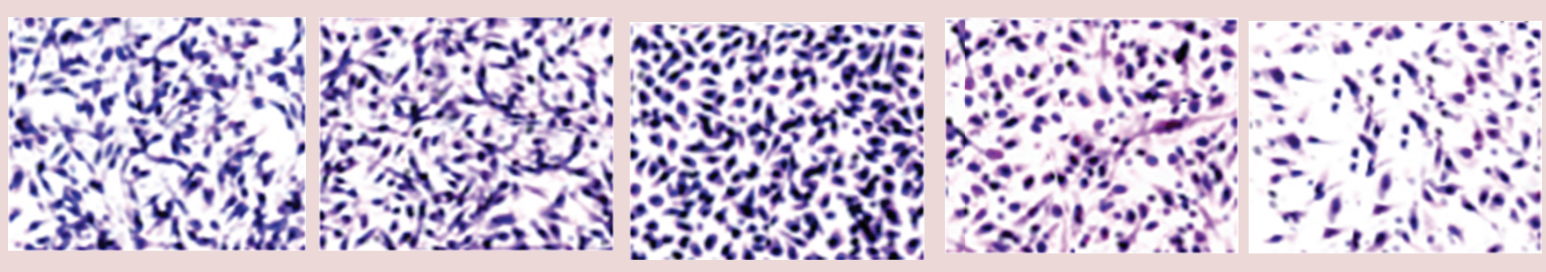

Figure 5. Anticancer effect of miRNA-130a and oxaliplatin in gastric cancer cells. (A) Apoptosis analysis of HGC27 cells by flow cytometer after staining with annexin- $\mathrm{V}$ and propidium iodide. The flow cytometer analysis was conducted with 10,000 cells as a mean count; (B) hoechst33342-based apoptosis analysis on the cancer cell morphology; (C) cell invasiveness of HGC27 cells and assessed by Matrigel transwell assays. The cells after incubation with different formulations for $24 \mathrm{~h}$, transferred to Transwell chambers and invading cells were observed under microscope.

miOXNP: Oxaliplatin and miR-130a-loaded lipid nanoparticles; OXL: Oxaliplatin; PD-miOXNP: PD-L1-conjugated oxaliplatin.

induced a remarkable $54 \%$ cell apoptosis, indicating the superior anticancer effect of the targeted formulations. The apoptotic effect was further confirmed by Hoechst staining (Figure 5B). Control cells were intact with perfect morphology, miR-130a and OXL treatment induced certain levels of apoptosis, and PD-miOXNP induced very high levels of apoptosis in cancer cells, as indicated by the high percentages of cell death, apoptotic body formation and condensation in cancer cells. The nuclei were not functional, and a large proportion of PD-miOXNP-treated cells were undergoing apoptosis, indicating its superior anticancer efficacy [27].

The anticancer effects of miR-130a and PD-miOXNP were established using western blot analysis, cell viability and cell apoptosis analyses. To examine the effects of these formulations on cell invasion, we conducted Matrigel invasion assays. In the nontreated control, entire cells invaded into the matrix, whereas miR-130a and OXL treatment groups showed slight reductions in cell invasion (Figure 5C). PD-miOXNP treatment showed the lowest invasion of cancer cells of any formulation, indicating the anticancer effect of the combinational treatment.

\section{The in vivo anticancer efficacy of PD-miOXNP}

The in vivo antitumor efficacy of different formulations was evaluated by intravenous administration of miR-130a, OXL, miOXNP and PD-miOXNP in the tumor xenograft model (Figure 6A). OXL was administered at a fixed dose of $5 \mathrm{mg} / \mathrm{kg}$ with the miR-130a dose fixed at $1.5 \mathrm{mg} / \mathrm{kg}$. As shown, blank NPs did not have any effect on 
(A)

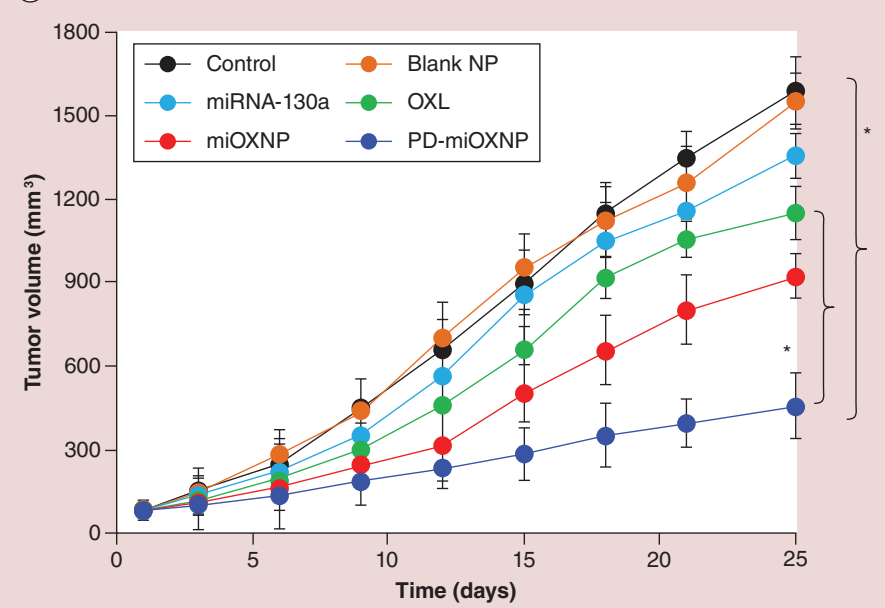

(B)

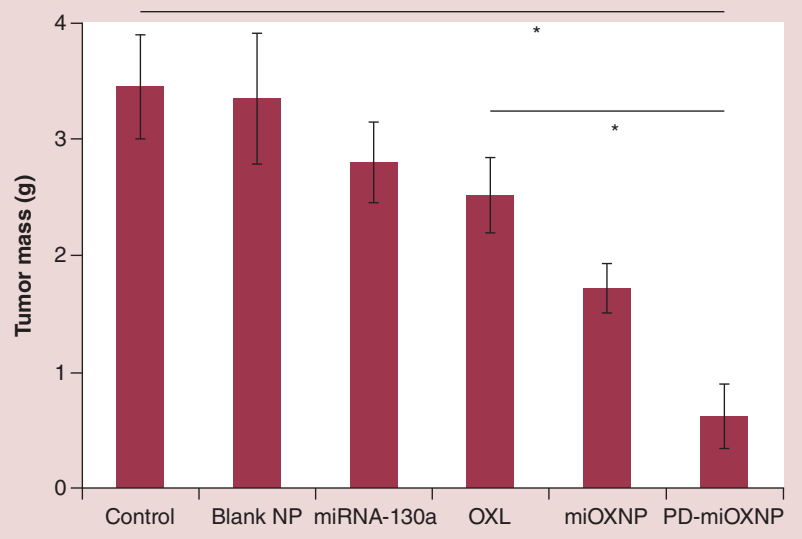

(c)

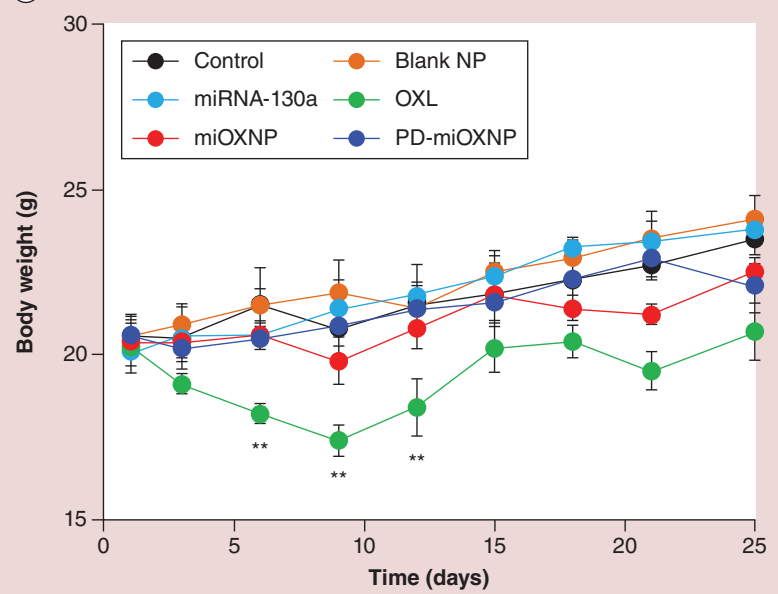

Figure 6. In vivo antitumor efficacy analysis in animal models. (A) In vitro antitumor efficacy analysis on xenograft nude mice after treatment with different formulations. The animals were treated with respective formulations for three-times and tumor volume was measured using vernier caliper. (B) Tumor mass analysis after extraction from the xenograft mice; (C) bodyweight analysis of mice after treatment with respective formulations. The bodyweight was monitored regularly until the study period.

${ }^{*} p<0.0001$ is the statistical difference of PD-miOXNP between OXL and control; ** $p<0.01$ is the statistical difference between PD-miOXNP and OXL.

miOXNP: Oxaliplatin and miR-130a-loaded lipid nanoparticles; NP: Nanoparticle; OXL: Oxaliplatin; PD-miOXNP: PD-L1-conjugated oxaliplatin.

tumor growth, and the tumor volume was equal to that of nontreated control. Free OXL and miR-130a, although initially effective, did not show much effect in controlling the tumor burden, and tumor growth progressed with time. A meaningful difference in tumor volume was observed for miOXNP compared with treatment with miRNA or OXL individually, indicating the synergistic effect of the therapeutic agents. Most importantly, PD-miOXNP showed significantly higher tumor growth inhibition than any other formulation. The final tumor volumes were approximately 1600, 1500, 1350, 1150, 900 and $450 \mathrm{~mm}^{3}$ for the control, blank NPs, miR-130a, OXL, miOXNP and PD-miOXNP treatments, respectively. Tumor weights are correlated with tumor volume, and showed the smallest tumor weight compared with any other formulations (Figure 6B).

The toxicity of the formulations was evaluated in terms of bodyweight analysis. Mice treated with free OXL lost more than $10 \%$ of their bodyweight compared with the nontreated control mice, indicating a severe systemic toxicity of free OXL (Figure 6C). In comparison, mice treated with OXL-loaded immunoliposomes did not show any signs of bodyweight loss, indicating the excellent safety of this carrier-based delivery system. 


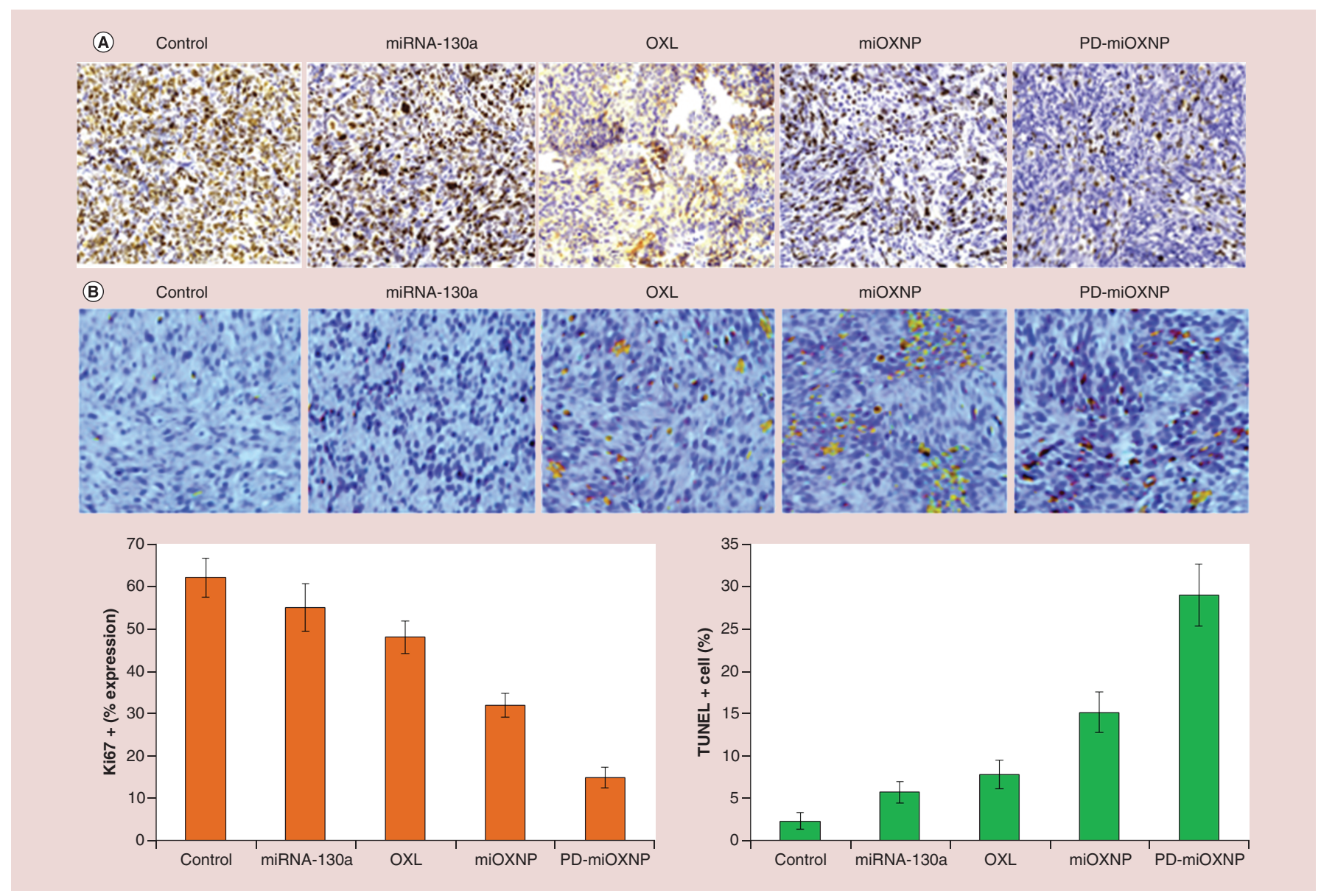

Figure 7. Immunohistochemical analysis of tumor sections from xenograft mice. (A) Ki67 proliferation marker analysis on the tumor section and (B) TUNEL staining of tumor section. The Ki67 and TUNEL assay were quantified and presented as a histogram. miOXNP: Oxaliplatin and miR-130a-loaded lipid nanoparticles; OXL: Oxaliplatin; PD-miOXNP: PD-L1-conjugated oxaliplatin; TUNEL: Terminal deoxynucleotidyl transferase-mediated nick end labeling.

\section{Immunohistochemistry analysis}

The mechanism of tumor cell death was further evaluated by immunohistochemical analysis. The expression of the Ki67 cancer cell proliferation marker was assessed. PD-miOXNP treatment induced a remarkable decrease in the number of $\mathrm{Ki} 7^{+}$cells compared with the nontreated control (Figure 7A). A significant difference in Ki67 ${ }^{+}$ cells was observed between PD-miOXNP and other formulations, further confirming the tumor growth findings. In addition, the apoptotic potential of the formulation was evaluated using the TUNEL assay (Figure 7B). Positive TUNEL results were barely detectable in tumors in mice treated with the control, whereas a few TUNEL-positive cells were observed after treatment with OXL and miR-130a. Significantly more TUNEL-positive cells were observed in tumors of mice treated with PD-miOXNP. The PD-miOXNP-treated cells were smaller and wider, with intracellular spaces and nuclear lysis, indicating inflammatory responses in the tumors. The dark brown color denoted apoptotic cells with condensed chromatin and lysed nuclear membranes. These results clearly indicate that PD-miOXNP was preferentially accumulated in gastric tumors and induced apoptosis and cell death through the synergistic action of miR-130a and OXL.

\section{Discussion}

GC is one of the most prevalent forms of cancer, with an estimated overall 5-year survival rate of merely $15 \%$ due to high rates of metastasis and recurrence. OXL is a third-generation platinum anticancer drug used in the treatment of GC. Although OXL possesses notable anticancer effects, single agent-based chemotherapy is increasingly ineffective, necessitating the development and use of combinational therapies. miR-130a has been shown to be involved in 
the pathogenesis of several cancers. We chose miR-130a as a potential oncomir targeting RAB5A and FOCL1 to improve GC treatment. Overall, the aim of the present study was to improve anticancer efficiency in GC by targeting combinational therapeutics (OXL and miR-130a) via PD-L1-targeted immunoliposomes. OXL was loaded into the lipid bilayers of the immunoliposomes, and miR-130a was loaded on the surface of NPs using the electrostatic interaction between miRNA and the positively charged liposome surface.

Nonspecific interactions between delivery carriers and nontargeted cells are always a critical challenge that may result in unnecessary side effects and at the same time decrease therapeutic efficacy during cancer treatment. PD-L1 expression is a valuable biomarker for cancer therapies that target immune checkpoints, which are expressed in several cancer cells. The US FDA has approved PD-L1-targeted mAbs for the treatment of different cancers. However, only some cancer patients responded to the antibody therapy due to differences in PD-L1 expression on different cancer cells. The results indicate that the high expression of PD-L1 receptors led to higher cellular uptake. A higher cellular uptake based on PD-L1 receptors should therefore increase the intracellular concentration of therapeutic agents, and a higher anticancer effect should occur [28]. Taken together, the results show that PD-L1-targeted immunoliposomes effectively enhanced cellular uptake when compared with nontargeted liposomes [29].

Western blot analysis was performed to determine the target specificity of miR-130a in GC cells. The results provided direct evidence that lower miR-130a expression contributed to the overexpression of FOSL1 and RAB5A and thereby promoted cancer cell growth. miR-130a directly targeted FOSL1 and RAB5A to suppress protein expression and cell growth. As shown, cell viability decreased significantly in a typical concentration-dependent manner with increasing concentrations of miR-130a. The results further confirmed the previous findings indicating the crucial role of FOSL1 and RAB5A in GC cell survival and growth. After observing the effect of miR-130a, the anticancer efficacy of combinational therapeutics was evaluated using the MTT assay. Treatment with the combination NPs (miOXNP) resulted in significantly lower cell viability than treatment with the free drug. More importantly, cells treated with PD-miOXNP showed significantly $(\mathrm{p}<0.01)$ lower cell viability than those treated with any other formulation, indicating that PD-L1 mAb-conjugated NPs targeted cells that overexpressed the PD$\mathrm{L} 1$ receptor/protein and exerted a remarkable anticancer effect. The PD-L1 mAb on the outer surface interacted with the respective receptors and resulted in enhanced cellular uptake and therapeutic efficacy. The results were consistent with the cellular uptake analysis, in which PD-L1-based carriers showed higher affinity toward the cancer cells compared with the carrier system without the PD-L1 ligand. Consistently, PD-miOXNP induced a remarkable $54 \%$ cell apoptosis, indicating the superior anticancer effect of the targeted formulations. In the same manner as cell viability, higher cellular uptake and higher intracellular concentration of PD-miOXNP may induce a higher level of apoptosis in cancer cells. PD-miOXNP treatment showed the lowest invasion of cancer cells of any formulation, indicating the anticancer effect of the combinational treatment. It should also be noted that OXL did not have much effect on controlling cell invasion, whereas there was a notable reduction in cell invasion in the miR-130a-treated group. Therefore, a combination of miR-130a and OXL significantly inhibited cell invasion.

Most importantly, PD-miOXNP showed significantly higher tumor growth inhibition than any other formulation. The remarkable antitumor efficacy of PD-miOXNP was mainly attributed to the specific tumor targeting ability of the PD-L1 mAb conjugated with the immunoliposomes. The PD-L1 mAb interacted with the PD-L1 receptor overexpressed in HGC27 tumors and likely resulted in enhanced intracellular concentration and enhanced antitumor efficacy. Most importantly, synergistic anticancer activity between OXL and miR-130a was the reason for the enhanced anticancer effect [30,31]. MiRNAs are small noncoding RNAs that play significant roles in cancer and can regulate multiple target genes, thus functioning as therapeutic molecules. Alterations in miRNA expression can modulate key cellular processes involved in tumorigenesis. miR-130a has been demonstrated as a key miRNA that controls diverse cellular processes including cell proliferation, apoptosis, autophagy, migration and angiogenesis. miR-130a targets the MET RTK and inhibits the proliferation of lung cancer cells [29]. miR-130a also targets ATG2B and DICER1 and inhibits leukemia cells [32]. Other targets of miR-130a include Rab5A, SMAD4 and HoxA5, resulting in the inhibition of multiple cancers [33-35]. Together, these results suggest that miR-130a may act as a tumor suppressor. A significant difference in $\mathrm{Ki}^{+} 7^{+}$cells was observed between PD-miOXNP and other formulations, further confirming the tumor growth findings and significantly more TUNEL-positive cells were observed in tumors of mice treated with PD-miOXNP.

The toxicity of the formulations was evaluated in terms of bodyweight analysis. As reported in other delivery systems, OXL had poor aqueous solubility and a short half-life in the blood, whereas nanocarriers improved its pharmacokinetic properties and thereby increased its therapeutic efficacy in cancer treatment. OXL is used as a first line of therapy in colorectal cancer and has been reported to suffer from severe liver damage. Besides, OXL has been 
reported to decrease the levels of white blood cells, lymphocytes and monocytes and cause myelosuppression. It is interesting to note that encapsulation of OXL in targeted liposome overcame the toxicity concerns of free OXL as indicated by no loss in the bodyweight $[36,37]$.

\section{Conclusion}

We designed and constructed PD-L1 mAb-conjugated miR-130a/OXL-loaded immunoliposomes for enhanced therapeutic efficacy against GC. The immunoliposomes showed good colloidal stability and released the encapsulated drug in a controlled manner under physiological conditions. HGC27 cells showed higher PD-L1 expression than MGC803 cells, indicating the specific targeting of HGC27 cells by immunoliposomes. The inhibitory role of miR-130a in GC cells was due to downregulation of the RAB5A and FOCL1 signaling pathways. Consequently, PD-miOXNP exhibited the strongest anticancer activity in vitro when compared with other formulations. The higher anticancer activity of miR-130a and PD-miOXNP was shown by cell viability, cell apoptosis and cell invasion analyses. In the in vivo xenograft model, PD-miOXNP exhibited significantly higher anticancer efficacy in HGC27 tumors with no systemic toxicity. Immunohistochemical analysis further showed reduced Ki67 ${ }^{+}$cells and increased TUNEL ${ }^{+}$cells in mice treated with PD-miOXNP, indicating its superior anticancer efficacy in animals. PD-L1 mAb-conjugated immunoliposomes, therefore, have potential utility for targeted therapy in PD-L1-positive GC patients, and could become a next-generation nanomedicine.

\section{Future perspective}

Immunoliposomes represent a new strategy that has attracted the attention of investigators worldwide owing to its application in preclinical cancer models with significant clinical trial results. The conjugation of various antibodies on liposomes presents an excellent opportunity for diversifying cancer applications. The combination of therapeutics with immunoliposomes in a single formulation may enhance the therapeutic potential of GC treatment in the future.

\section{Summary points}

- PD-L1 monoclonal antibody-conjugated miR-130a/oxaliplatin-loaded immunoliposomes exhibited enhanced therapeutic efficacy against gastric cancer.

- PD-L1-conjugated oxaliplatin particles (PD-miOXNP) maintained stability without being significantly affected by the environment for fairly long periods of time.

- HGC27 cells showed remarkably higher PD-L1 expression than MGC803 cells.

- miR-130a directly targeted FOSL1 and RAB5A to suppress protein expression and cell growth.

- PD-miOXNP showed significantly $(p<0.01)$ lower cell viability and higher apoptosis than any other formulation.

- PD-miOXNP showed significantly higher tumor growth inhibition in mice than any other formulation.

- PD-miOXNP induced a remarkable decrease in the number of $\mathrm{Ki}^{+} 7^{+}$cells and increase in $\mathrm{TUNEL}^{+}$cells.

- PD-L1 monoclonal antibody-conjugated immunoliposomes show great potential as a treatment for PD-L1-positive gastric cancers.

\section{Open access}

This work is licensed under the Attribution-NonCommercial-NoDerivatives 4.0 Unported License. To view a copy of this license, visit http://creativecommons.org/licenses/by-nc-nd/4.0/

Author contributions

F Wang was responsible for all the drug-loaded formulations and synthesis. Y Sun was responsible for all the in vitro and animal experiments. J Shi has designed the experiment, secured the grant and analyzed and written the entire manuscript.

Financial \& competing interests disclosure

This study was supported by Public Welfare Project of Zhejiang Science and Technology Hall (PWPZSTH; grant no: 2017C33130; 2017F30045). The authors have no other relevant affiliations or financial involvement with any organization or entity with a financial interest in or financial conflict with the subject matter or materials discussed in the manuscript apart from those disclosed.

Writing support was provided by TEXTCHECK and was funded from the financial assistance of PWPZSTH (grant no: 2017F30045) 


\section{Ethical conduct of research}

All animal and experimental procedures were approved by Animal Care and Use Committee of Tongde Hospital of Zhejiang Province, China. The authors state that they have obtained appropriate institutional review board approval or have followed the principles outlined in the Declaration of Helsinki for all animal experimental investigations.

\section{References}

Papers of special note have been highlighted as: • of interest; $\bullet \bullet$ of considerable interest

1. Jemal A, Bray F, Center MM, Ferlay J, Ward E, Forman D. Global cancer statistics. CA Cancer J. Clin. 61, 69-90 (2011).

2. Isobe $\mathrm{Y}$, Nashimoto A, Akazawa K et al. Gastric cancer treatment in Japan, annual report of the JGCA nationwide registry. Gastric Cancer 14, 301-316 (2011).

3. Ferlay J, Soerjomataram I, Dikshit R et al. Cancer incidence and mortality worldwide: sources, methods and major patterns in GLOBOCAN. Int. J. Cancer 136, E359-E386 (2015).

4. Schwarz RE, Zagala-Nevarez K. Recurrence patterns after radical gastrectomy for gastric cancer: prognostic factors and implications for postoperative adjuvant therapy. Ann. Surg. Oncol. 9, 394-400 (2002).

5. Cappetta A, Lonardi S, Pastorelli D, Bergamo F, Lombardi G, Zagonel V. Advanced gastric cancer (GC) and cancer of the gastro-oesophageal junction (GEJ): focus on targeted therapies. Crit. Rev. Oncol. Hematol. 81, 38-48 (2012).

- Due to a comprehensive presentation of all the targeted approaches.

6. Sundaramoorthy P, Ramasamy T, Mishra SK et al. Engineering of caveolae-specific self-micellizing anticancer lipid nanoparticles to enhance the chemotherapeutic efficacy of oxaliplatin in colorectal cancer cells. Acta Biomater. 42, 220-231 (2016).

- Due to ability to target the gastric cancers (GCs).

7. Alcindor T, Beauger N. Oxaliplatin: a review in the era of molecularly targeted therapy. Curr. Oncol. 18(1), 18-25 (2011).

- Due to a potential approach to target the GCs using immunonanoparticles.

8. Boll K, Reiche K, Kasack K et al. MiR-130a, miR-203 and miR-205 jointly repress key oncogenic pathways and are downregulated in prostate carcinoma. Oncogene 32, 277-285 (2013).

9. Kovaleva V, Mora R, Park YJ et al. miRNA-130a targets ATG2B and DICER1 to inhibit autophagy and trigger killing of chronic lymphocytic leukemia cells. Cancer Res. 72, 1763-1772 (2012).

10. Li L, Zhou L, Li Y, Lin S, Tomuleasa C. MicroRNA-21 stimulates gastric cancer growth and invasion by inhibiting the tumor suppressor effects of programmed cell death protein 4 and phosphatase and tensin homolog. J. BUON. 19(1), 228-236 (2014).

11. Zheng B, Liang L, Wang C et al. MicroRNA-148a suppresses tumor cell invasion and metastasis by downregulating ROCK1 in gastric cancer. Clin. Cancer Res. 17(24), 7574-7583 (2011).

- One of the first study to highlight the importance of miR-148a in GCs.

12. Zhang J, Wu H, Li P, Zhao Y, Liu M, Tang H. NF-kappaB-modulated miR-130a targets TNFalpha in cervical cancer cells. J. Transl. Med. 12, 155 (2014).

13. Zhang X, Huang L, Zhao Y, Tan W. Downregulation of miR-130a contributes to cisplatin resistance in ovarian cancer cells by targeting X-linked inhibitor of apoptosis (XIAP) directly. Acta Biochim. Biophys. Sin. (Shanghai) 45, 995-1001 (2013).

14. Qiu S, Lin S, Hu D, Feng Y, Tan Y, Peng Y. Interactions of miR-323/miR-326/miR-329 and miR-130a/miR-155/miR-210 as prognostic indicators for clinical outcome of glioblastoma patients. J. Transl. Med. 11, 10 (2013).

15. Kovaleva V, Mora R, Park YJ et al. miRNA-130a targets ATG2B and DICER1 to inhibit autophagy and trigger killing of chronic lymphocytic leukemia cells. Cancer Res. 72, 1763-1772 (2012).

16. Chen X, Zhao M, Huang J et al. MicroRNA-130a suppresses breast cancer cell migration and invasion by targeting FOSL1 and upregulating ZO-1. J. Cell Biochem. 119(6), 4945-4956 (2018).

17. Ramasamy T, Ruttala HB, Gupta B et al. Smart chemistry-based nanosized drug delivery systems for systemic applications: a comprehensive review. J. Control. Rel. 258, 226-253 (2017).

$\bullet \quad$ It is a detailed presentation of drug delivery approach in cancer treatment.

18. Sundaramoorthy P, Baskaran R, Mishra SK et al. Novel self-micellizing anticancer lipid nanoparticles induce cell death of colorectal cancer cells. Colloids Surf. B Biointerfaces 135, 793-801 (2015).

19. Rodallec A, Brunel JM, Giacometti S et al. Docetaxel-trastuzumab stealth immunoliposome: development and in vitro proof of concept studies in breast cancer. Int. J. Nanomedicine 13, 3451-3465 (2018).

- It explores using an immunoliposome as a potential carrier.

20. Brown KC. Peptidic tumor targeting agents: the road from phage display peptide selections to clinical applications. Curr. Pharm. Des. 16(9), 1040-1054 (2010).

- Due to the clinical applications.

21. Adams GP, Weiner LM. Monoclonal antibody therapy of cancer. Nat. Biotechnol. 23(9), 1147-1157 (2005). 
22. Brahmer J, Reckamp KL, Baas P et al. Nivolumab versus docetaxel in advanced squamous-cell non-small-cell lung cancer. $N$. Engl. J. Med. 373(2), 123-135 (2015).

23. Garon EB, Rizvi NA, Hui R et al. Pembrolizumab for the treatment of non-small-cell lung cancer. N. Engl. J. Med. 372(21), 2018-2028 (2015).

24. Robert C, Long GV, Brady B et al. Nivolumab in previously untreated melanoma without BRAF mutation. N. Engl. J. Med. 372(4), 320-330 (2015).

25. Pardoll DM. The blockade of immune checkpoints in cancer immunotherapy. Nat. Rev. Cancer 12(4), 252-264 (2012)

26. Ramasamy T, Haidar ZS, Tran TH et al. Layer-by-layer assembly of liposomal nanoparticles with PEGylated polyelectrolytes enhances systemic delivery of multiple anticancer drugs. Acta Biomater. 10, 5116-5127 (2014).

27. Ruttala HB, Chitrapriya N, Kaliraj K et al. Facile construction of bioreducible crosslinked polypeptide micelles for enhanced cancer combination therapy. Acta Biomater. 63, 135-149 (2017).

28. Liang SC, Latchman YE, Buhlmann JE et al. Regulation of PD-1, PD-L1, and PD-L2 expression during normal and autoimmune responses. Eur. J. Immunol. 33(10), 2706-2716 (2003).

29. Acunzo M, Visone R, Romano G et al. MiR-130a targets MET and induces TRAIL-sensitivity in NSCLC by downregulating miR-221 and 222. Oncogene 31, 634-642 (2012).

30. Ramasamy T, Ruttala HB, Sundaramoorthy P et al. Multimodal selenium nanoshell-capped Au@mSiO2 nanoplatform for NIR-responsive chemo-photothermal therapy against metastatic breast cancer. NPG Asia Materials 10, 197-216 (2018).

31. Tran TH, Thapa RK, Nguyen HT et al. Combined phototherapy in anti-cancer treatment: therapeutics design and perspectives. J. Pharm. Invest. 46, 505-517 (2016).

32. Kovaleva V, Mora R, Park YJ et al. miRNA-130a targets ATG2B and DICER1 to inhibit autophagy and trigger killing of chronic lymphocytic leukemia cells. Cancer Res. 72, 1763-1772 (2012).

33. Lee SH, Jung YD, Choi YS, Lee YM. Targeting of RUNX3 by miR130a and miR-495 cooperatively increases cell proliferation and tumor angiogenesisin gastric cancer cells. Oncotarget 6, 33269-33278 (2015).

34. Liu Y, Li Y, Wang R et al. miR-130a-3p regulates cell migration and invasion via inhibition of Smad4 in gemcitabine resistant hepatoma cells. J. Exp. Clin. Cancer Res. 35, 19 (2016).

35. Pan Y, Wang R, Zhang F et al. MicroRNA-130a inhibits cell proliferation, invasion and migration in human breast cancer by targeting the RAB5A. Int. J. Clin. Exp. Pathol. 8, 384-393 (2015).

36. Yang Q, Cai J, Sun S et al. Polymer nanoparticle delivery of dichloroacetate and DACH-Pt to enhance antitumor efficacy and lower systemic toxicity. Biomater. Sci. 4(4), 661-669 (2016).

37. Tummala S, Kumar MN, Pindiprolu SK. Improved anti-tumor activity of oxaliplatin by encapsulating in anti-DR5 targeted gold nanoparticles. Drug Deliv. 23(9), 3505-3519 (2016). 